\title{
Research on electronic control system of heavy vehicle multi-axle
}

\section{steering}

\author{
Shengrui Liu
}

\author{
Automotive Engineering institute, Jiangxi University of Technology, Nanchang 330098, China
}

Keywords: Electronic control system; Heavy vehicle; Multi-axle steering

\begin{abstract}
The vehicle steering performance directly affects the vehicle's flexibility handling stability and economy for use. By changing the wheel angle of the axles except the front axle, the multi-axle steering technology improves the vehicle's flexibility and mobility at low speed when turning and improves the vehicle's handling stability at high speed. In this paper, the research on the electronic control of the heavy vehicle multi-axle steering system is conducted. Because the system has relatively more tasks and stronger real-time property, the C/OS-II operating system is applied here. In this paper, based on the study of the fundamental theory, the mathematical analysis and modeling are implemented on the electronically controlled hydraulic actuator. The software MATLAB is applied to simulate the mathematical model of execution system so as to verify its validity.
\end{abstract}

\section{Introduction}

In recent years, due to the sharp increase in cars, road traveling space becomes less and less. All kinds of vehicles tend to develop towards the multi-axle steering system, especially large engineering vehicles which often bring bad conditions to the road. The requirements of Multi Axle Steering are extremely urgent. The vehicle steering performance directly affects the vehicle's flexibility handling stability and economy for use. By changing the wheel angle of the axles except the front axle, the multi-axle steering technology improves the vehicle's flexibility and mobility at low speed when turning and improves the vehicle's handling stability at high speed. The instantaneous over-steering or under-steering of the vehicle is changed to enhance the dynamic stability of the vehicle, thus making cars be safer and avoid being out of control due to oversteer or understeer. With the development of the electronic technology especially the applied electronic control technology, the automobile is rapidly becoming a carrier integrating mechatronics and various high technologies, enabling the automobile to develop towards high reliability, automation and intelligent vehicle. Multi axle steering research has also been considerably developed. These results are attributed to the application of the computer control unit (ECU).

The development of ECU product is a system engineering, which is closely related to the mechanical system, sensor, actuator, chip, connectors, shell etc. Due to special requirements, some parts often need to be customized, involving the complex process of designing hardware and software. With the rapid development of automobile electronic technology, more and more tasks need ECU to process. Faced with these problems, the old-fashioned design method of ECU software has been unable to meet the needs. The embedded multi-task real-time operating system is the best way to solve this problem. The research of embedded real-time multi task operating system has decades of history. There are also researchers who study the embedded system for automotive 
electronic control, but it is for the control of the body of small-size car, such as window lift, light switch etc. The relevant research on the embedded real-time multi task operating system heavy vehicles and other large vehicles multi axle steering control technology are still blank. From the perspective of the development of science and technology and the automotive industry, the application of embedded multi task real-time operating system in this area is urgently needed. The heavy vehicles studied in this paper have characteristics of high quality, large inertia, high centroid, large wheelbase and numerous axles, whose requirement of achieving high flexibility and trafficability is very urgent. The multi axle steering technology has a significant effect on improving the heavy vehicle's handling stability at high speed and low-speed maneuverability so that heavy vehicles can enter the very narrow field to work.

Although there are multi axle steering vehicle products in foreign countries, because of technology secrecy and patent issues, searchable documentation is very limited. Foreign multi axle steering technology has reached a very high level, which can conduct $4 \sim 9$ independent bridge steering and can well improve the steering performance of heavy-duty vehicles. The domestic multi axle steering technology is still in the initial stage. There is a big gap to its foreign relative. Although there are a few heavy vehicles equipped with multi axle steering system, it is only designed for low-speed maneuverability but cannot improve the vehicle's handling stability in normal driving condition.

Steering system control mode can be divided into mechanical, hydraulic and electronic multi axle steering according to its structure and actuator. At present the multi axle steering device has been combining the mechanical, hydraulic, electronic, sensor and microprocessor control technology closely, which forms electronically controlled hydraulic multi axle steering system, mainly composed of sensors, electronic control unit, control valve, hydraulic cylinder etc.

C/OS-II is a complete, portable, curing, cutting the preemptive real-time multitasking kernel. C/OS-II is written in ANSI C language, containing a small amount of assembly language code, which can be used for different microprocessor. The following are parts of the concept of C/OS-II in the paper.

(1) task (2) multi tasks (3)task switching (4) kernel (5) scheduling (6) signal (7) message queue

With the development of computer network technology, the network technology that can be applied to vehicles is increasing, whose function is powerful, but because of the rapid development of industry, the tasks that must be finished in the actual construction is more and more complex. This requires that ECU which controls the machine can handle more complex tasks, so the old fashioned ECU software design method is inadequate and even cannot meet the needs of the design. the embedded real-time multi task operation system is chosen to solve this problem.

This paper is aimed to the research on the electronic control part of heavy vehicle multi axle steering system. Because the system has numerous tasks and strong real-time property, the C/OS-II operating system is adopted. In this paper, based on the study of the fundamental theory, the mathematical analysis and modeling are implemented on the electronically controlled hydraulic actuator. The software MATLAB is applied to simulate the mathematical model of execution system so as to verify its validity. The paper designs the software and hardware of electronic control system. Under laboratory conditions, the hardware simulation and software debugging are implemented to design the circuit board. 


\section{Methods}

In this paper, the electronic control system is divided into two parts: the main control module and execution module. The main control module is the control center of the whole control system, which is to collect the value of the front wheel angle and the value of speed, to adopt the control strategy of this laboratory (zero sideslip angle control strategy) to calculate the rear axle angle to obtain the theoretical steering angle value and pass the value to the execution module. The execution module is the "nerve of execution" of the electronic control system, which is to receive the theoretical steering angle value of the main control module, to process the value and output to the actuator, and to control the rear wheel to turn at a actual angle through the actuator. The execution module collects the rear wheel actual angle value from feedback loop. Compare it with the theoretical angle value. Use the results of the comparison to modify the actual angle value. Pass the modified value to the actuator to decrease the error between the actual rear wheel angle value and the theoretical one until the error is within the allowable range.

The essence of PID control is to base the calculation on the function relationship between the proportion, integral and differential according to the input deviation value, and to control the actuator by using the calculation results for the output. It has a simple structure and its parameters easy to adjust and has accumulated rich experience in the long-term application, especially in industrial process control. Because it is difficult to establish an accurate model of the controlled object and the system parameters often change, if modern control theory analysis is used, it will take a very high price for model identification and often can not reach the expected effect. However, the use of PID control and the online parameter setting based on experience can achieve satisfactory control effect. Besides, with the development of the computer especially microcomputer technology, digital PID control algorithm can be achieved in a single chip machine. Because of the flexibility of the software system, the PID algorithm can be modified and improved. Although the modern control theory has been rapidly developed in modern industrial production, the vast majority of control loop is still using the PID controller which is simple and reliable and is easy to realize and steady-state error free, etc.

Electric control system mainly consists of main control module and execution module, involving signal acquisition unit (including the speed signal, the signal of steering angle, rear axle angle signal), and that the core component is the sensor signal acquisition; calculation unit (variables are speed and front wheel angle, namely, the steering wheel angle); signal output unit (completed by execution module); feedback unit (mainly use displacement sensor to send the displacement of the hydraulic cylinder value back to the execution module ). Here is the concrete control procedure. The main control module acquires the driving speed and steering wheel angle; calculate the value of the rear axle wheel angle according to multi axle steering control strategy (zero side slip angle proportional control strategy) to; the execution module receives the rear axle angle sent by the main control module; through the PID control and then the PWM transformation to control the size of the opening of the proportional electromagnetic so as to control the displacement of the hydraulic cylinder to control the angle of the rear axle; meanwhile use the sensor to measure the rear axle angle value, then send the measured value back to the execution module, and through the PID control output error value to correct the steering angle, so as to achieve the goal of precise control. It can be seen from the above analysis that the sensor unit is the precursor and standard of this design, which is unique and important. Usually, as for automotive sensors, a large number of parameters that the vehicle needs to monitor and control are non-constant power, so the sensor is the "eyes" of auto control 
system. Whether its choice is correct or not will directly affect the quality of monitoring and control of the various parts of the car, thus affecting the overall performance of the car. The selection of sensors can mainly be done from the following aspects: measuring range, sensitivity, resolution, error (including repeat error), linearity, overload capacity, reliability, response time etc.

The main control module for rear axle angle calculation needs to collect two parameters: the speed and angle of the front wheel. The sensor selected to measure the speed value in the design is Holzer speed sensor, which will transform the speed of transmission output shaft into a sequence pulse with different frequency. Then the single chip machine counts the impulse sequence and transforms it into the current speed. But in the process of transmission, larger capacitance on the transmission line will cause the rising edge of the waveform to go bad obviously; long transmission line and the impedance of the transmission line not matching that of the receiver will cause vibration on the rising and falling edges of the waveform. There are some other reasons such as external disturbances that will make rectangular pulse generate waveform distortion. For these effects, Schmidt reverse trigger can be used to obtain the relatively ideal rectangular pulse waveform. The positive feedback in a Schmidt trigger transition process can make periodic signal of the edge that changes slowly as the rectangular pulse signal. If the input signal is greater than the VT+, we can get the rectangular pulse signal with same frequency in the output end of the Schmidt trigger. As long as VT+ and VT- of the Schmidt trigger are set appropriately, the satisfactory cosmetic effects can be obtained.

The software design of the electronic control system is an indispensable part of the whole system design. It connects control law, the control algorithm and the hardware resources together to realize the control function. In this paper, the software of the electronic control system includes system software and application software or the main control module and the execution module. Because the main control module completes more tasks, an ordinary single program can not meet the needs, so in the main control module C/OS-II embedded real-time multi task operating system is treated as the system software to control specific tasks whose functions can be written in C51 language. In the execution module, the tasks to be completed are relatively small, so the application software is written in ASM51 microcontroller assembly language.

With the rapid development of electronic technology, modern industrial production has formed a complete system integrating design, simulation, experiment, production. The simulation of the whole system plays an important role, which not only can test the accuracy of the design, but also provides a reference for physical experiment. The continuous improvement of the software makes the simulation results of the software more accurate. In the design and production, the application of the software not only accelerates the design speed and improves work efficiency, but also can greatly reduce the design cost. Obviously, in the future development and production of the actual product, extensive use of software is a big trend. This paper simulates the hardware circuit, and simulation software is a famous circuit simulation software MultiSim8. MultiSim8 is a circuit simulation software with powerful circuit simulation function, launched by a Canadian technology company (Interactive Image Technoligics , or IIT company).

After conducting software simulation for the electronic control system and debugging the software and hardware under laboratory conditions, the design of the circuit board of the system is begun. PCB (printed circuit board) is the bridge from a circuit diagram to plating. That is, before plating, PCB board should be designed first. In this paper the design of PCB board adopts ProtelDXP circuit board design software launched by Altium Company, which provides enough working layer, 
embedded with a large number of advanced design rules required for manufacturing and producing, thus freeing the user from the complex circuit design rules

\section{Conclusion}

The multi axle steering technology is the key technology in the development of large construction vehicles. The domestic multi axle steering technology is still at the initial stage. Compared with foreign countries there is a big gap. Starting from designing a set of electronic control system with excellent performance and high reliability, by reading a large number of domestic and foreign research on heavy vehicle multi axle steering system and referring to a large amount of related information about electronic control system, the paper puts forward a steering system driven by the electric hydraulic and based on embedded multi task operating system C/OS-II, and designs the hardware and software of the electronic control system. The main contents are summarized as follows:

(1) This paper analyzes the research status of domestic and foreign automobile multi axle steering, and does an in-depth study on the embedded multi task operating system C/OS-II. As for the requirements of flexible low-speed steering and stable high-speed of the plentiful multi axle heavy vehicles, combining the characteristics of the current electronic control system, the paper puts forward the method of using embedded multi task operating system C/OS-II timely to implement macro control of the individual task of the multi axle steering system to fulfill the demand of being swift and stable.

(2) This paper establishes the transfer function from the amplifier circuit output voltage to the rear axle of hydraulic cylinder displacement and illustrates the feasibility of this transfer function for the simulation analysis of the mathematical model under the Simulink environment of the Matlab software; it introduces in details the conventional PID control strategy and digital PID control strategy, and combine the control characteristics of electronic control system to select the incremental PID control strategy to control the steering axle.

(3) It chooses hardware equipment of the electronic control system, including: sensor, controller and other components, and designs the signal acquisition circuit based on the signal output form of the sensors (including: speed sensor signal acquisition circuit, steering wheel angle signal acquisition circuit, rear axle angle signal feedback circuit), ECU control unit (including: the processing circuit of the signal and the peripheral circuit of the microcontroller) and the driving circuit of the proportional valve etc.

(4) This paper designs the software part of the main control module. First, transplant C/OS-II to AT89S52 microcontroller, and then taking C/OS-II as a platform, use single-chip C language to write the main module software program. Considering the characteristics of the few tasks in execution module, the software program of the execution module is written in assembly language.

(5) It adopts the MultiSim8 software to simulate the peripheral circuit of the control system, which shows the validity of the peripheral circuit of the control system; implement the joint debugging of the system's hardware and software under laboratory conditions; in addition, it uses Protel DXP software to design the system circuit board. 


\section{Acknowledgements}

This work was financially supported by the key subject building project (vehicle engineering) of Jiangxi University of Technology.

\section{References}

[1] Palkovics L, Fries A. Intelligent electronic systems in commercial vehicles for enhanced traffic safety[J]. Vehicle System Dynamics, 2001, 35(4-5): 227-289.

[2] CHEN N, YIN G, LI P, et al. Control of four-wheel steer vehicle maneuver and developing platform of hardware-in-loop-simulation [J][J]. Journal of Jiangsu University (National Science Edition), 2005, 5.

[3] He Y, Islam M M, Webster T D. An integrated design method for articulated heavy vehicles with active trailer steering systems[R]. SAE Technical Paper, 2010.

[4] LIU L, LUO Y, LI K. Tire-road friction coefficient estimation based on normalized tire model[J]. Journal of Tsinghua University (Science and Technology), 2009, 5: 025.

[5] Watanabe K, Yamakawa J, Tanaka M, et al. Turning characteristics of multi-axle vehicles[J]. Journal of Terramechanics, 2007, 44(1): 81-87.

[6] Li Y, Yang L, Yang G. Network-based coordinated motion control of large-scale transportation vehicles[J]. Mechatronics, IEEE/ASME Transactions on, 2007, 12(2): 208-215.

[7] Li Y, Yang L, Yang G. Network-based coordinated motion control of large-scale transportation vehicles[J]. Mechatronics, IEEE/ASME Transactions on, 2007, 12(2): 208-215.

[8] Jin S H U. Analysis of the Motion for Four Wheel Steering Vehicle With Proportional Control [J][J]. Special Purpose Vehicle, 2002, 3: 16-19.

[9] Pu Q Y C N L. Direct yaw-moment control on four-wheel steering vehicles [J][J]. Journal of Southeast University (Natural Science Edition), 2004, 4: 006.

[10] Sakurai H, Ono M, Mamiya A. Electric vehicle: U.S. Patent 4,913,258[P]. 1990-4-3. 